Cuadernos de Filología Italiana

ISSN: 1133-9527

http://dx.doi.org/10.5209/CFIT.56036

\title{
Remo Ceserani: La confianza en los estudiantes
}

Mirella Marotta ${ }^{1}$

Recibido: 03 de mayo de 2017 / Revisado: 02 de agosto de 2017 / Aceptado: 21 de agosto de 2017

Resumen. En esta breve contribución se pasa revista a la obra del crítico desaparecido Remo Ceserani, analizando los conceptos que han sido la guía a lo largo de su amplia producción. El primero entre todos, el concepto de imaginario, a partir del cual propuso una nueva forma de entender la literatura y la crítica literaria. Se analiza también su especial concepción de lo fantástico. Por último, se pone de relieve su gran confianza en el futuro y en los estudiantes.

Palabras clave: Literatura italiana; crítica literaria; imaginario; literatura fantástica.

[en] Remo Ceserani: The trust in the students

\begin{abstract}
This short contribution provides an outlook on the already missing critic Remo Ceserani, focusing on the concepts that have been his guidelines along his wide production. Among them and first of all, the concept of imaginary, based on which he proposed a new way to understand Literature and its Critics. This paper analyses as well his special understanding of the fantastic. Finally, the paper highlights Ceserani's trust on the future and the students.
\end{abstract}

Key words: Italian Literature; Literary Criticism; The Imaginary; The Fantastic.

Sumario: 1. Una nueva óptica en los estudios literarios 2. El imaginario 3. Lo fantástico 4. Nuestros gigantes

Cómo citar: Marotta, Mirella (2017): «Remo Ceserani: La confianza en los estudiantes», Cuadernos de Filología Italiana, 24, pp. 227-237.

\section{Una nueva óptica en los estudios literarios}

En 1996 Remo Ceserani fue invitado a participar con uno de sus trabajos en esta misma revista del Departamento de Filología Italiana de la Universidad Complutense; era el número 3 de Cuadernos de Filología Italiana y el crítico italiano, ahora desaparecido, publicaba su trabajo sobre «L'apparente armonia dell'Orlando Furioso». En su artículo analizaba el hecho de que la sensibilidad de los lectores actuales encuentra dificultades para enfrentarse a la poesía de Ariosto, que la crítica de principios del siglo XX había establecido como paradigma de escritura canónica, perfecta, había exaltado su clasicismo y, sobre todo, su armonía; pero todas estas cualidades la convertían en algo demasiado sublime, prácticamente inaprehensible.

Ciudad Universitaria s/n. 28040 Madrid.

marotta@ucm.es 
Sin embargo, continuaba Ceserani, la crítica más actual «dopo aver sottoposto a nuove analisi l'opera intera di Ariosto, e in particolare il Furioso, ne ha scoperto la reale disarmonia e la forte irrequietezza di fondo, recuperandola, anche per questa via, al gusto moderno» (Ceserani 1996: 128). Con esta premisa, el trabajo continuaba analizando la obra del escritor ferrarés vista a la luz de la nueva óptica, a partir de la cual Ceserani nos descubría más y nuevos valores, más y nueva belleza en la producción ariostesca.

He querido empezar este trabajo sobre la ingente producción de Remo Ceserani y sobre lo mucho que ha dejado a los estudiosos, los estudiantes y la crítica literaria posterior, partiendo de este artículo con el que honró a nuestra revista porque creo que es una muestra de lo que hizo durante toda su vida: ver la literatura y la crítica desde otra óptica, liberarse de los cánones establecidos, de lo que se entendía habitualmente que eran los estudios literarios y buscar otro punto de vista, otra forma más actual y más sugerente de estudiar y enseñar la literatura.

Alumno de Fubini en la Statale de Milán y posteriormente ayudante suyo en la Normale de Pisa, Ceserani inició su carrera como crítico literario con la publicación de ediciones de los grandes clásicos italianos, Decameron, Orlando furioso, Canzoniere de Petrarca, Poesie de Carducci; pero al mismo tiempo se ocupó también de editar o traducir a autores de otras culturas: D. H. Lawrence, Joyce, Zola... Desde el inicio de su actividad estuvo convencido de que la literatura no podía estudiarse de forma aislada dentro de unas fronteras lingüísticas o nacionales, como tampoco podía comprenderse sola, como algo áulico y autónomo, sino que debía ser vista en el conjunto de las artes y las ciencias, como parte de ellas. Uno de sus libros menos conocidos, y sin embargo, al menos desde mi punto de vista, una obra clave para la fijación de las bases de esta nueva perspectiva, ha sido Convergenze. Gli strumenti letterari e le altre discipline. Lo publicó en 2010, casi al final de su carrera y, de alguna forma, se aprecia en él un punto de síntesis, una comprensión madura y convencida de la relación indisoluble entre la literatura y las demás actividades del conocimiento humano, al tiempo que de aquello que la literatura aporta a las otras disciplinas.

Todo el texto es una defensa de los estudios interdisciplinares y una demostración de la imposibilidad de conocer partiendo de casillas aisladas. Ceserani hace suyas las palabras de David Cooper, profesor de la Universidad de Oakland, en favor de los estudios interdisciplinares:

Secondo lui (Cooper) ci sono due ragioni principali:

1) La ricerca interdisciplinare costruisce ponti fra le singole discipline o ristruttura il sistema delle discipline.

2) L'interdisciplinarietà corregge l'eccesso di specializzazione. (Ceserani 2010: 7)

Y el crítico continúa citando a Cooper y defendiendo una serie de razones que, a su juicio, avalan la necesidad de no centrarse en estudios meramente literarios o lingüísticos sino de abrir el campo a las otras ciencias: son razones de carácter epistemológico — «la ricerca della conoscenza e della verità è servita meglio con 1'aiuto degli studi interdisciplinari»_-, de carácter ético - un trabajo interdisciplinar privilegia la unidad y la integración como bienes dignos de ser perseguidos- - de carácter estético — «c'è piacere nella sintesi e anche bellezza nei processi che producono simmetria e interezza», de carácter político y, por supuesto, de carácter pedagógico: solo a través de la exposición de argumentos distintos ofrecidos por varias discipli- 
nas se puede aspirar a ofrecer una educación que potencie el pensamiento crítico y la empática (Cesarini 2010: 8).

Al mismo tiempo Ceserani se pregunta qué es lo que la literatura aporta a las demás disciplinas y considera que la respuesta está en dos elementos característicos del texto literario y de su organización retórica: «l'uso funzionale della metafora e il ricorso diffuso alla narrazione» (Ceserani 2010: 12). Por lo que al primer aspecto se refiere, sostiene que se ha producido un fuerte cambio de perspectiva en la distinción tradicional neta entre discurso científico y discurso poético, cambio que tiene que ver precisamente con el hecho de reconocer que el uso de la metáfora ha constituido un ingrediente esencial del lenguaje de la química, de la biología o de la medicina desde sus orígenes, que los científicos denominaron las categorías de la naturaleza creando metáforas sobre la forma, función o estructura de cada ser o de cada elemento. Sobre el papel que tuvo en este proceso el uso de las formas de la narración, aporta opiniones de psicólogos, médicos, antropólogos para proponer y defender una relación estrecha entre las formas de la consciencia y las formas de la narración, entre los diversos modos de describir, juzgar, interpretar el mundo y las formas de la representación literaria.

En las páginas sucesivas analizará la relación del lenguaje de la literatura y de sus formas con la filosofía, las matemáticas, la física, la química, la biología, la historia, la economía, la medicina, la neurociencia... para concluir que, después de haber indagado de forma tan metódica en la interdisciplinariedad, lo que ha encontrado en todas las disciplinas es un interés por los problemas éticos de la vida individual y colectiva. Esta conclusión lo llena de satisfacción y optimismo en el futuro.

En su obra Guida breve allo studio della letteratura, pone como premisa a todo el trabajo la idea de que podemos señalar dos tendencias contrapuestas en los estudios sobre literatura:

La de quienes defienden su autonomía y su especificidad con respecto a cualquier otra forma de comunicación humana, y la de quienes tienden, en cambio, a relativizar toda idea de literatura y a ponerla en contacto con las distintas formas de comunicación existentes, que pueden variar en función de la óptica y del contexto cultural. (Ceserani 2003 [2004: 2])

A continuación expone de forma sistemática los argumentos que sostienen una y otra teoría, decantándose, como era evidente, por la segunda. Esto último lo hace sobre todo a partir de un argumento: «La literatura es una entre las muchas manifestaciones del imaginario humano y posee afinidades con otras formas y otros modos de simbolización y transcripción cultural de la realidad» (Ceserani 2003 [2004: 5]). Estamos ya ante su concepto más querido, el que guió como un faro toda su actividad y toda su carrera, a cuya luz nos propuso una nueva forma de interpretar el arte: el imaginario.

\section{El imaginario}

No se pretende hacer en estas páginas una revisión de toda la obra de Ceserani y, desde luego, no se pretende hacerlo de forma cronológica; no obstante, resulta evidente que casi se ha empezado por el final. Y sin embargo, son los últimos escritos 
del crítico los que nos desvelan con más claridad lo que se propuso durante toda su carrera. Esa exaltación de los estudios interdisciplinares había sido, desde el primer momento, el motor que lo había llevado a cambiar la forma en la que explicaba la literatura. En una entrevista del 2013, en la que se le preguntaba sobre qué había cambiado en Italia en la escuela y en los estudios literarios, Ceserani respondía:

Non mi pare che sia cambiato molto. È in atto da anni una tendenza, in molti paesi e anche in Italia, a trascurare nell'educazione scolastica gli insegnamenti delle cosiddette materie letterarie (la cura della lingua, la ricchezza e le sfumature del linguaggio parlato e scritto, lo spessore della storia), così come di quelli delle scienze forti (la matematica, le scienze naturali, i metodi della ricerca), e a dedicarsi molto di più alle conoscenze pratiche e strumentali o ad apprendimenti molto superficiali. Spesso si contrappongono, nelle discussioni fra pedagogisti e insegnanti, scienze umane e scienze naturali. In realtà le scienze naturali sono spesso trascurate al pari delle scienze umane. Io che mi occupo di letteratura, sono pronto a sostenere con forza la necessità di un forte incremento delle conoscenze scientifiche (fisica, biologia, studio delle piante e degli animali). Non va dimenticato che molti scienziati e filosofi sono stati grandi scrittori, da Galileo a Darwin, da Schopenhauer a Leopardi, da Nietzsche a Freud. Lo sapeva benissimo Calvino. E sono pronto a sostenere la grande importanza, nella formazione sia individuale sia collettiva, delle varie forme dell'immaginario (quindi anche dell'immaginario letterario) per affrontare la vita sempre più complessa delle nostre società. (Sismondini 2013: 213)

Es, por tanto, el imaginario, entendido en toda su extensión, aplicado a todos los campos del saber, el que aúna el conocimiento. A partir de esta idea y de la constatación de su extraordinaria trascendencia, Ceserani concibió una nueva forma de presentar la materia literaria y de enseñarla a los estudiantes. Nace de allí Il materiale e l'immaginario, el manual de literatura y de cultura que creó junto a una profesora del Liceo Gioberti de Turín, Lidia De Federicis. Esta obra revolucionó la escuela, la forma de enseñar, y continúa hoy en día aportándonos un modelo de presentación del mundo en el que vivimos más completo y más global. Los 10 volúmenes que lo componen no son solo una antología de literatura o una enciclopedia de las ciencias humanas, son sobre todo, en palabras de Pierluigi Pellini, pronunciadas en memoria del maestro poco después de su muerte en la Sala Azzurra de la Scuola Superiore Normale di Pisa, «una sfida alla curiosità e all'intelligenza di insegnanti e studenti, un serbatoio inesauribile di suggerimenti di lettura, un'immagine della cultura finalmente lontana da ogni provincialismo autoritario, da ogni pigra certezza manualistica» (Pellini 2016: s/p).

El germen de la obra fue una serie de artículos que Ceserani había publicado en la revista Belfagor. La directora de la casa editorial Loescher, Maria Laura Gardoncini, lo llamó; le dijo que conocía bien las críticas que él había escrito sobre los manuales de literatura y que le proponía hacer uno que mejorara lo existente hasta ese momento en el mercado. Ceserani respondió que lo haría con una única condición: que Lidia De Federicis entrara a formar parte de la empresa. En diversas ocasiones el maestro ha contado cómo fue a Turín a ver a Lidia, cómo charlaron durante días en la buhardilla en la que ella tenía su estudio y cómo, sin perder un momento, pusieron 
manos a la obra. Inicialmente se proyectaron tres volúmenes para los tres años de la escuela superior, pero en seguida se dieron cuenta de que la empresa era mucho más importante y que no podía pararse: así Il materiale e l'immaginario apareció en 8 volúmenes, más dos adicionales de Manuale.

En la entrevista ya mencionada que le hizo Alberto Sismondi, Ceserani explicaba lo que había pretendido con esta obra:

Come lei sa io ho allestito a suo tempo, insieme con la compianta Lidia De Federicis, un'antologia, Il materiale e l'immaginario (Torino, Loescher) che ha proposto un modo nuovo di affrontare l'insegnamento della letteratura, mettendola in rapporto con le altre forme del sapere (dalla vita materiale a quella delle idee), allargando la scelta dei testi ben oltre i confini nazionali e affiancando ai grandi testi classici altri testi, per varie ragioni interessanti. Abbiamo anche cercato, il più possibile, di opporci alla tradizione scolastica italiana dell'antologia (la scelta dei brani famosi, il fior da fiore), proponendo per ogni anno almeno la lettura di due o tre testi completi (a questo scopo davamo tutta una serie di strumenti di supporto critico della lettura) e spesso accompagnando le letture con richiami all'integrità del testo, con proposte di allargamento e confronto con altri testi. Il progetto, nato in anni di grandi speranze culturali (vere e proprie utopie), ha avuto per un certo numero di anni uno straordinario successo, presso una classe di insegnanti motivati ma anche di studenti curiosi (ne incontro ancora adesso molti, che mi dicono di aver trovato in quel libro grandi stimoli per la loro formazione). (Sismondini 2013: 215)

La enciclopedia literaria, que se propuso como un «laboratorio di analisi e di lavoro critico», cambió la forma de estudiar literatura y probablemente también la forma de ver el mundo. En el momento de su muerte, Ceserani estaba haciendo una revisión de toda la materia, enfocada a una reedición de la obra que incluyera actualizaciones sobre muchos temas que habían cambiado desde el 1978, año en que apareció el primer volumen. Maria Laura Gardoncini (2016), en el discurso en homenaje al crítico en ocasión de su muerte, informaba de este trabajo de revisión, en el que Ceserani estaba incluyendo todos los avances en instrumentos informáticos y multimedia, llevado, como siempre, por su inagotable curiosidad y su fe en la enseñanza. Termina diciendo la directora de Loescher que la casa editorial no dejará el trabajo inacabado, que publicará de nuevo Il materiale e l'immaginario aunque ya no estén ninguno de los dos autores que lo crearon.

Para Romano Luperini (2013: 1) esta gigantesca obra era, antes que nada, un laberinto, pero un laberinto ordenado y estructurado, una especie de inmenso catálogo y de gran enciclopedia del imaginario. Sus 10 volúmenes comparten con la enciclopedia de la Ilustración el optimismo y la carga innovadora y reformadora que da la confianza en la educación de los jóvenes.

Me parecen muy acertadas las palabras de Luperini y me doy cuenta de que, de alguna forma, yo he tenido muchas veces la sensación de que había algo de «roussoniano» en Il materiale e l'immaginario. La afirmación es, cuanto menos, arriesgada y debo concretarla; cuando hablo del eco de Rousseau en sus volúmenes, me refiero al deseo de formar, de enseñar, de hacer madurar (esto por encima de todo) a los estudiantes; y me refiero también a una fe en ellos, en sus capacidades y sus poten- 
cialidades, superior a la de cualquier otro autor o crítico contemporáneo. Esta es la mayor aportación de Ceserani, desde mi punto de vista, el ser capaz de sustraerse a la desazón habitual ante los cambios y ante el sistema educativo y pensar que las nuevas generaciones no son menos capaces ni menos inteligentes de lo que lo eran las anteriores, que, en su nueva forma de acercarse al lenguaje, a los textos a la información, al mundo, hay una brillantez muy especial.

Prácticamente todos los que han escrito sobre él en estos meses, desde su desaparición han resaltado esta extraordinaria cualidad. Pierluigi Pellini, por ejemplo, habla de la dedicación con la que Ceserani se había entregado en los últimos tiempos a la revisión de Il materiale e l'immaginario, lo estaba haciendo «con l'entusiasmo giovanile e quasi incosciente che non lo aveva mai abbandonato, con la sbalorditiva capacità di lavoro che tutti gli invidiavamo, con la sua incrollabile fiducia nell'intelligenza dei giovani» (Pellini 2016: s/p).

Alberto Sismondini, en la entrevista que hace al crítico en diciembre de 2013, le pregunta por la educación de los jóvenes y si cree que la literatura sigue siendo un arma imprescindible en la enseñanza en las escuelas medias y superiores. Ceserani reconoce que hay motivos para la preocupación tanto por la burocratización de la educación como por la desmotivación de muchos docentes; sin embargo, en seguida su discurso cambia y se vuelve alegre y esperanzado, llegando incluso a hablar de posibilidades que mueven al entusiasmo: el entusiasmo está para él siempre ligado a los jóvenes, a los estudiantes:

Remo: (...) È vero d'altra parte che, soprattutto nei cicli della scuola elementare e media inferiore, se gli insegnanti sono bravi e hanno un vero senso della lingua e del ritmo della poesia, delle capacità metaforiche e inventive del linguaggio, della precisione e chiarezza di un discorso ben strutturato, possono trovare un riscontro entusiasmante negli alunni, i quali amano istintivamente giocare sia con le immagini sia con le parole e hanno un gusto nativo per la narrazione. (Sismondini 2013: 214)

Y lo mismo podemos encontrar en una larga conversación de Ceserani con dos colegas, profesoras ambas de la escuela media, Orsetta Innocenti y Simona Micali; ellas conocen la pasión del crítico por los jóvenes y saben de antemano que sus respuestas van a ir siempre en esa dirección, pero - llevadas por las dificultades en el trabajo que desarrollan día a día - no pueden sino dar su visión pesimista de un sistema educativo que presenta muchos problemas; y no solo se quejan de lo institucional, se quejan también de que los jóvenes leen menos, pasan horas ante el ordenador, tienen una capacidad de expresarse y de memorizar menor de la que se tenía en generaciones anteriores. Ceserani no cede en su opinión positiva de las nuevas generaciones, acepta que hay un empobrecimiento de los lenguajes verbales, pero «hanno anche nuove forme di intelligenza e di capacità comunicativa rapide, flessibili, spesso creative» (Innocenti e Micali 2013: 8). En las páginas de la larga conversación entre los tres profesores, que tiene lugar mientras caminan a lo largo de la Piagge, un paseo arbolado de unos dos kilómetros a orillas del Arno, encontramos una y otra vez el mismo planteamiento: las quejas de ellas a lo difícil que resulta el papel de docente en esta sociedad, que se topan con la respuesta decidida de Ceserani sobre lo mucho que tienen que enseñarnos a nosotros las nuevas generaciones de estudiantes. Ellas siguen exponiendo la situación con toda su dureza, y él: 
Adesso la situazione che abbiamo appena descritto della scuola, degli insegnanti, degli incerti interventi ministeriali rende l'impresa ancora più rischiosa. L'unico dato che può aprire qualche spiraglio di speranza è quello che abbiamo detto parlando dei giovani, del loro rapporto con la rete, fatto di disinvoltura, ma anche intelligenza e una dose nativa di creatività, Paradossalmente il Materiale e l'immaginario, nato quando la rete era solo agli albori del suo sviluppo (pensate quanto le cose siano cambiate in così poco tempo), sembrava fatto apposta per il futuro sviluppo della rete. (Innocenti e Micali 2013: 13)

Las palabras de Ceserani no pueden ser más expresivas y nos demuestran que, si a lo largo de su labor como crítico y como formador se propuso dar un nuevo punto de vista a los estudios literarios y a la manera de enseñarlos, era porque sabía que la mentalidad de los estudiantes había cambiado, que necesitaban acercarse a los textos de otra forma, pero sobre todo, y esto es lo más importante, porque estaba persuadido de que esta nueva forma era mejor. El paseo por el Arno se cierra con una preciosa metáfora con la que el crítico mira al futuro y ve en él una esperanza y la decidida necesidad de seguir luchando:

Remo: Vi prego di seguire con lo sguardo lo scorrere lento dell'Arno, che a volte, per effetto della marea, sembra addirittura andare contro corrente. Là in fondo, vedete, ora che le sere scendono ormai prima di quanto avveniva d'estate, si vede il cielo colorarsi dei colori del tramonto. Più che il filo rosso di cui parlavi tu, Orsetta, vediamo come un orizzonte rosso. Come lo interpretiamo? Come un segnale apocalittico o come un segnale di speranza? Come un simbolo di eclettismo, di relativismo, di rifiuto di ortodossie filosofiche e religiose, di laicità? Nella parola d'ordine che avete lanciato, il richiamo alla laicità, io mi riconosco totalmente. Lidia, la mia amica Lidia, dopo le delusioni politiche e sindacali, si era tutta dedicata, con l'irruenza che le era propria, al movimento per la difesa della laicità, insieme con molti altri insegnanti, magistrati, scienziati torinesi. Io, nelle rubriche che scrivo per Aracne, per La ricerca e per L'immaginazione batto continuamente su questo chiodo. Per questo tenderei a interpretare il rosso di questo tramonto in chiave se non di speranza almeno di impegno fattivo. (Innocenti e Micali 2013: 17)

\section{Lo fantástico}

No sé si sería correcto decir que el imaginario lleva a Ceserani directamente hasta lo fantástico. Quizá la afirmación es demasiado simplista y necesita muchos más matices, pero lo que sí es cierto es que el crítico partió de un concepto tan querido para dar una nueva perspectiva, en este caso, al género de literatura fantástica. Siguiendo su estructura argumentativa habitual, señala dos corrientes críticas contrapuestas: la de quienes consideran lo fantástico como modo literario concreto y lo reducen poco más que a unos cuantos textos de la literatura del Romanticismo europeo (entre estos autores coloca a Todorov con su conocidísima definición del género fantástico); y, en segundo lugar, la de quienes buscan ensanchar el campo de acción de lo fantástico, sacarlo de los límites históricos de un movimiento y un siglo concretos, abrirlo a 
todo un sector de la producción literaria que abarca otros modos narrativos, formas y géneros. Obviamente, Ceserani se sitúa entre estos últimos y utiliza (esta vez sí) el concepto de imaginario para hacer entrar dentro del género desde el cuento heroico al cuento popular, desde la novela utópica a la de terror a la de ciencia ficción, hasta llegar incluso a la novela de ocultismo o la apocalíptica.

El extraordinario capítulo segundo, «Tentativi di definizione», ofrece una revisión de las teorías sobre el género de los autores más significativos. Parte de Todorov, como no podía ser de otra manera, del que, a pesar de dejar claro que tienen visiones contrapuestas sobre el tema, exalta su extraordinaria definición de lo fantástico como lapso de tiempo de vacilación: «Lo fantástico es la vacilación que experimenta un ser que sólo conoce las leyes naturales, ante un acontecimiento aparentemente sobrenatural.» (Todorov 1970 [1980: 28]). Pero aporta también muchas otras definiciones muy interesantes, como la de Pierre George Castex, Roger Caillois, entre otros. En 2001 el Museo Romántico de Madrid me pidió un estudio sobre la literatura fantástica en Italia; en esa ocasión tomé partido por las teorías de Todorov y titulé el trabajo que se publicó en la Revista del Museo Romántico, «Del lapso de vacilación en la literatura romántica italiana»; sin embargo, visto ahora desde la perspectiva de los años, mi aproximación a la materia bebía más del planteamiento de Ceserani que del de Todorov.

Pero el capítulo realmente sobresaliente e innovador de Il fantastico es, desde mi punto de vista, el tercero, «Procedimenti formali e sistemi tematici del fantastico» en el que hace un magistral catálogo de los procedimientos retóricos y estilísticos que son característicos del género; es decir nos ofrece una guía completa y pormenorizada de todo aquello que, una vez detectado en un texto, nos indica que podemos estar ante una forma de relato fantástico. Pero no se detiene en lo meramente formal; pasa a continuación a recoger también elementos temáticos propios de estas obras, desde el temor o lo tenebroso - que resultan obvios - a otros motivos menos previsibles como el sueño, el doble o el viaje, entendidos como «passaggi di soglia e di frontiera»:

Abbiamo spesso incontrato, nei racconti fantastici che abbiamo letto, esempi di passaggio dalla dimensione del quotidiano, del familiare e del consueto a quella dell'inesplicabile e del perturbante: passaggi di soglia, per esempio, dalla dimensione della realtà, a quella del sogno, dell'incubo o della follia. Il personaggio protagonista si trova d'improvviso come dentro due dimensioni diverse, con codici diversi a disposizione per orientarsi e capire. (Ceserani 1996: 80)

Este paso de lo cotidiano a lo inesperado, lo inexplicable, que tiene lugar dentro de un texto aparentemente normal, nos sitúa directamente en la esfera de lo posmoderno. Diversos críticos han encontrado en este trabajo de Ceserani un concepto nuevo, que han bautizado como Neofantástico. A partir de él es posible interpretar la obra de autores como Calvino, Cortázar o Bioy Casares, pero no solo, también otros como Celati, Tabucchi o Baricco, en los que la irrupción de lo inesperado y casi imposible es con mucha frecuencia el punto de partida de la narración. La última parte de mi actividad docente e investigadora la he dedicado a enseñar y a profundizar sobre la obra de estos tres últimos autores y constantemente tengo que volver al mil veces abierto y releído trabajo de Ceserani. Él analiza solo un cuento de Tabucchi, I pomeriggi del sabato, en el que el protagonista narrador, un niño de diez o doce 
años, tiene un contacto con lo sobrenatural, con el fantasma de su padre; el niño no lo ve nunca, pero siente su presencia con total nitidez. La perspectiva interesante de este cuento, como de gran parte de la literatura de la posmodernidad, es que no hay sorpresa ni incredulidad; lo inexplicable se acepta como un dato más, como el «rovescio del reale» que constituye el primer elemento en la poética de Tabucchi y que encontramos también en los otros escritores que hemos mencionado. Por dar dos breves ejemplos de esto que hemos bautizado Neofantástico, se podría citar el cuento de Gianni Celati, «Vivenza di un barbiere dopo la morte», en el que un barbero ignorado por su mujer y por las demás personas del pequeño pueblo de Piacenza en el que vive, llega a la conclusión de que todos le niegan la existencia, y de que esto está ligado al hecho de que, durante la guerra, un soldado alemán le disparó a las orillas del Trebbia sin llegar a alcanzarlo, pero que, obviamente, todos piensan que lo mató y por ese motivo actuan de esa forma excluyente. Además, después de que se haya encontrado en la escalera de su casa a un amigo que hace tiempo que ha fallecido, la mujer lo repudia alegando que está loco. El cuento concluye:

Alcuni mesi dopo la morte del barbiere sua moglie si è scoperta incinta, e ha sparso la voce che aspettava un figlio del barbiere morto. In seguito ha anche sparso la voce che il barbiere le aveva parlato di notte, dicendosi molto contento che lui avesse riconosciuto il figlio come suo, perché così aveva smesso di negargli l'esistenza. (Celati 1985: 40)

Y se podría citar también uno de los últimos libros publicados por Alessandro Baricco, Tre volte all 'alba, que el propio autor presenta con la nota que sigue, nota que coloca dentro de la narración, desde el planteamiento inicial, en la esfera de lo imposible, de la física cuántica y sus universos paralelos o simplemente, en la concepción de Ceserani, de lo fantástico:

Queste pagine raccontano una storia verosimile che, tuttavia, non potrebbe mai accadere nella realtà. Raccontano infatti di due personaggi che s'incontrano per tre volte, ma ogni volta è l'unica, e la prima, e l'ultima. Lo possono fare perché abitano un Tempo anomalo che inutilmente si cercherebbe nell'esperienza quotidiana. Lo allestiscono le narrazioni, di tanto in tanto, e questo è uno dei loro privilegi. (Baricco 2012: 7)

\section{Nuestros gigantes}

El otro gran trabajo que el crítico nos ha dejado fue Raccontare il postmoderno, el libro en el que "dialoga" con los escritores y los intelectuales más importantes de finales del siglo XX, muchos de ellos grandes amigos suyos, como Fredric Jameson o Umberto Eco, pero, por encima de todo, en el que desmontaba esa especie de leyenda negra que perseguía en Italia a la nueva generación de escritores que la vieja y consolidaba generación despreciaba por no considerarla suficientemente impegnata. Ceserani pone de manifiesto los valores de la nueva forma de escribir y de ver la 
realidad y los defiende frente a lo viejo con la misma decisión que en Il materiale e l'immaginario había defendido los valores de los jóvenes. Romano Luperini dice al respecto:

Per lui il postmoderno doveva essere, anzi sicuramente era, «uno dei più grandi cambiamenti, epocali e totali, che si siano avuti nella storia dell'umanità» (come si legge nella Introduzione a La ricerca letteraria e la contemporaneità, nono volume del Materiale e dell'immaginario). (Luperini 2013: 4)

Tenemos muchos otros textos inolvidables, Treni di carta del 1993, donde analiza la evolución de la sociedad a la luz de las nuevas tecnologías en materia de viaje y transportes; o uno de los últimos, L'occhio della medusa: fotografia e letteratura (2011), en el que retorna a lo que aprendió de su padre, fotógrafo de profesión, para ver nuevamente el imaginario, esta vez en la relación entre literatura e imagen instantaneizada. Ahora solo nos queda seguir consultando las innumerables páginas que nos ha dejado escritas e intentar aprender de ellas. En este sentido, quisiera recoger un texto que aparece en la página web del departamento de Derecho Procesal de la Universidad Complutense sobre «Universidad y excelencia verdadera» (2016). En él, Andrés de la Oliva Santos, catedrático de ese departamento, busca el origen de una frase que ha sido citada por muchos y que parece tener un origen mucho más antiguo de lo que se pensaba, se remonta al siglo XII y Andrés de la Oliva la atribuye a Bernardo de Chartres:

En el siglo XII, justo cuando nacían las Universidades, vivió, trabajó y escribió un Bernardo de Chartres, filósofo neoplatónico, canciller de la Catedral de Chartres, al que se debe una frase redonda, que en siglos posteriores se atribuyó a otros personajes (a Isaac NewTon, por ejemplo), cuando, en realidad, ya JUAN DE SAlisbury, en su obra Metalogicon, de 1159, citaba con toda claridad a Bernardo. Bernardo de Chartres decía que «somos como enanos a hombros de gigantes. Podemos ver más, y más lejos que ellos, no por la agudeza de nuestra vista ni por la altura de nuestro cuerpo, sino porque alcanzamos lo alto levantados por la altura gigantesca

Ceserani, con su deseo inagotable de replantearse la literatura, con su forma abierta de entender la cultura y el mundo en el que se inserta, con su fe en los estudiantes y en sus capacidades para hacer que este mundo vaya adelante, será para siempre unos hombros sobre los que subirnos.

Quisiera citar como cierre a estas páginas en memoria del crítico otro breve texto suyo, el que cierra Convergenze:

Forse posso anch'io ripetere, con voce sommessa, quello che scriveva da Princeton Clifford Geertz una decina di anni fa prima di lasciarci per sempre - anche perché anch'io, come lui, preferisco le volpi ai Ricci, gli ardimenti ottimisti ai catastrofisti per partito preso, gli amanti del disordine e della confusione a quelli che vorrebbero tenere in un modo tutto bene ordinato e incasellato-: «Tempi 
interessanti [quelli che ci aspettano], invidio coloro che stanno per ereditarli». (Ceserani 2010: 169)

\section{Referencias bibliográficas}

Baricco, Alessandro (2012): Tre volte all'alba, Milano Feltrinelli.

Celati, Gianni (1985): «Vivenza di un barbiere dopo la morte», en Gianni Celati, Narratori delle pianure, Milano Feltrinelli, pp. 38-40.

Ceserani, Remo (1993): Treni di carta. L'immaginario in ferrovia: l'irruzione del treno nella letteratura moderna, Torino, Bollati Boringhieri.

Ceserani, Remo (1996): «L'apparente armonia dell'Orlando Furioso», Cuadernos de Filología Italiana, 3, pp. 125-143.

Ceserani, Remo; De Federicis, Lidia (1979-1982): Il materiale e l'immaginario: Laboratorio di analisi di testi e di lavoro critico, (10 vol.) Torino, Loescher.

Ceserani, Remo (1996): Il fantastico, Bologna, Il Mulino.

Ceserani, Remo (1997): Raccontare il postmoderno, Torino, Bollate Boringhieri.

Ceserani, Remo (2003), Guida breve allo studio della letteratura, Roma, Laterza. [Introducción a los estudios literarios, Barcelona, Crítica S.L., 2004]

Ceserani, Remo (2010): Convergenze. Gli strumenti letterari e le altre discipline, Milano, Mondadori.

Ceserani, Remo (2011): L'occhio della medusa: fotografia e letteratura, Torino, Bollati Boringhieri.

Gardoncini, Maria Laura (2016), «Il materiale e l'immaginario», Oltre il ponte, 2 de noviembre de 2016. <http://www.oltreilponte.org/cultura/_il-materiale-e-1_immaginario/>

Innocenti, Orsetta; Micali, Simona (2013): «Insegnamenti. Passeggiando alle Piagge con Remo Ceserani, Between, III.6. <http://www.Between-journal.it/>

Luperini, Romano (2013), «Ceserani e la scuola», Between, III.6. <http://www.Betweenjournal.it/>

Marotta, Mirella (2001): «Del lapso de vacilación en la literatura romántica italiana», Revista del Museo Romántico 3, pp. 97-102.

Oliva de la, Andrés (2016): «Universidad y excelencia verdadera», <http://www.ucm.es/ dpto-derechoprocesal/texto-prof-dr-andres-de-la-oliva-santos $>$

Pellini, Pierluigi (2016): «Per Remo Ceserani», Le parole e le cose, 5/11/1916, http://www. leparoleelecose.it/?p=24945

Sismondini, Alberto (2013) «Entrevista a Remo Ceserani», Revista de Estudios Literarios, 3, Universidad de Coimbra, pp. 213-221.

Todorov, Tzvetan (1970): Introduction à la littérature fantastique, Paris, Seuil. [Introducción a la literatura fantástica, Méjico, Premia, 1980]. 